livraisons

d'Histoire

de l'Architecture

\section{Livraisons de l'histoire de l'architecture}

$30 \mid 2015$

Le dessin d'architecture : œuvre/outil des architectes

$?$

\title{
La querelle du décor : aspects et évolutions de l'architecture intérieure des musées
}

Dispute over decor. Features and developments of museum interior design

Der Dekorstreit. Aspekte und Entwicklungen der Innenarchitektur von Museen

\section{Yannick Le Pape}

\section{OpenEdition}

Journals

Édition électronique

URL : http://journals.openedition.org/lha/557

DOI : 10.4000/lha.557

ISSN : 1960-5994

\section{Éditeur}

Association Livraisons d'histoire de l'architecture - LHA

\section{Édition imprimée}

Date de publication : 30 décembre 2015

Pagination : 105-115

ISSN : 1627-4970

Référence électronique

Yannick Le Pape, « La querelle du décor : aspects et évolutions de l'architecture intérieure des musées », Livraisons de l'histoire de l'architecture [En ligne], 30 | 2015, mis en ligne le 18 décembre 2017, consulté le 03 juin 2020. URL : http://journals.openedition.org//ha/557 ; DOI : https://doi.org/10.4000/ Iha.557 


\section{Varia}


Par Yannick LE PAPE

\section{LA QUERELLE DU DÉCOR : ASPECTS ET ÉVOLUTIONS DE L'ARCHITECTURE INTÉRIEURE DES MUSÉES}

En arrivant au musée, les œuvres d'art ne font pas qu'intégrer une collection. Elles intègrent aussi un nouvel environnement architectural et décoratif avec lequel elles vont devoir composer. Que le bâtiment soit lui-même chargé d'histoire complique certes les choses, comme en témoignait Georges Salles, l'ancien directeur du Louvre, en 1950, lorsqu'il constatait que la disposition des sculptures au sein du musée devait être pensée « en rapport avec les volumes des salles, leurs formes, leur perspectives et leurs styles ${ }^{1}$. " Le décor du musée, en d'autres termes, représenterait une véritable contrainte, sans que cela, d'ailleurs, ne soit nécessairement préjudiciable : ainsi André Chastel souligna-t-il combien « Michel Laclotte a fort heureusement tiré parti, avec ses décorateurs, du cadre du $\mathrm{XV}^{\mathrm{e}}$ siècle $^{2}$ " à l'occasion de la création du musée du Petit Palais, à Avignon. Le décor offert aux collections royales, à la fin du XVIII ${ }^{\mathrm{e}}$ siècle, avait déjà suscité le débat, avec une préférence clairement exprimée pour "la salle du Louvre qui par son étendue et la magnificence de son architecture est la plus belle pièce qu'il y ait dans aucun palais du Roy ${ }^{3}$ ". Les visiteurs, du reste, ne s'y trompaient pas: lorsque le révérend Dawson Warren visita le Museum national en novembre 1801, il ne manqua pas de mentionner non seulement les collections mais aussi les "salles magnifiques ${ }^{4}$ " qui les accueillent.

\section{Indigence, exubérance et mauvais goût: le décor à l'amende}

La pauvreté en matière décorative fut parfois source d'inquiétude, comme en 1931, lorsqu'on évoqua l'installation du musée du costume dans le château de Sceaux : le Bulletin des musées de France rapporte à cette occasion que "les plus ardents promoteurs du futur musée l'avaient accepté malgré ses inconvénients évidents :

1. Georges Salles, Au Louvre. Scènes de la vie du musée, Paris, Domat, 1950, 250 p., p. 85-86.

2. André Chastel, "Dans les murs de Jules II ", André Chastel, Le présent des ceuvres, Paris, Fallois, 1993, 280 p., p. 72.

3. Voir Geneviève Bresc-Bautier, "La salle des Antiques du palais du Louvre ", Jean-Luc Martinez (ed.), Les Antiques du Louvre. Une histoire du goût d'Henri IV à Napoléon I'r , Paris, Fayard, 2004, 240 p., p. 67-78.

4. Cité par Jean Galard dans Promenades au Louvre: en compagnie d'écrivains, d'artistes et de critiques d'art, Paris, Robert Laffont, 2010, 1231 p., p. 86. 
éloignement du centre parisien, exiguïté relative des locaux, médiocrité du décor ${ }^{5}$ ". Philippe de Chennevières, directeur des Beaux-Arts, rapportait dès 1883 combien le président Mac-Mahon avait été indisposé par la banalité des salles que le Louvre consacrait aux antiquités ${ }^{6}$. On peut aussi se remémorer ce que disait Prosper Mérimée dans la Revue des deux mondes au sujet du Salon Carré tel qu'il se présentait avant 1848: "Chacun s'est demandé si des Raphaëls et des Titiens devaient être suspendus sur des murailles mal crépies et s'il était décent d'exposer tant de trésors dans une salle qui, pour la décoration, ressemblait fort à une écurie ${ }^{7}$ ! ".

La solution, dès lors, est peut-être d'intervenir sur le décor. Les efforts en la matière lors de la création du pavillon Denon ${ }^{8}$, au Louvre, montrent que l'option était connue. Encore faut-il faire l'unanimité dans ce domaine. Viollet-le-Duc ne cacha pas son scepticisme quant aux murs recouverts de tentures colorées du British Museum ${ }^{9}$ : "J'irais à Londres [...] que je n'irais pas voir les bas-reliefs du Parthénon [...] et que si je les voyais dans leur trou actuel, je ne pourrais plus me les figurer qu'entourées de rideaux verts ${ }^{10}$. " Manuel Escriva de Romani, du Prado, condamnait de la même manière en 1936 les pinacothèques du XIX ${ }^{\mathrm{e}}$ siècle, aux murs rougeâtres : "Dans certains cas, écrivait-il, le caprice du décorateur s'emparait d'un motif archaïque dépourvu de toute raison d'être et qui n'était qu'un échantillon [...] du mauvais goût du moment ${ }^{11}$."

Le Rijksmuseum conçu par Pierre Cuypers, à la fin du XIX ${ }^{\mathrm{e}}$ siècle, subit les mêmes reproches. David C. Röell, directeur du musée entre 1945 et 1959, s'exprimait à ce sujet lors de la rénovation de 1955: "Ce bâtiment, qui date de 1885, était certes digne d'éloges du point de vue des proportions et de l'éclairage. Mais, fils de son temps, Cuypers y avait multiplié les ornements et les inscriptions sans toujours se soucier de leur opportunité, et construit des salles inutilisables rappelant les styles d'autrefois ${ }^{12}$." (ill. 1) Les choix de Félix Duban pour la décoration du Louvre, lorsqu'il prit la suite de Pierre-François-Léonard Fontaine en 1848, n'avaient pas fait non plus l'unanimité. "Ainsi, regrettait Gustave Planche, la décoration du

5. P. V., "Le Musée du costume, à Fontenay-aux-Roses ", Bulletin des Musées de France, $\mathrm{n}^{\circ}$ 10, octobre 1931, 16 p., p. 222-223.

6. Philippe de Chennevières, Souvenirs d'un directeur des Beaux-Arts, première partie, Paris, Aux bureaux de "L'Artiste ", 1883, 111 p., p. 53.

7. Cité par Christiane Aulanier dans Histoire du palais et du musée du Louvre. Le Salon Carré, Paris, Réunion des musées nationaux, 1950, 85 p., p. 65.

8. Voir Pierre Quoniam (dir.), Le Louvre. Sept visages d'un musée, Paris, Réunion des musées nationaux, 1986, 367 p., p. 19.

9. Une carte postale satirique de 1822 (Tom and Bob in search of the Antique, British Museum, CE115/2/31/1) atteste de cet aménagement.

10. Voir Bruno Foucart (éd.), Viollet-le-Duc. L'éclectisme raisonné, Paris, Denoël, 1984, 335 p., p. 161163.

11. Manuel Escriva de Romani, "Les principes de la décoration intérieure des musées de Madrid ", Mouseion, vol. 31-32, Paris, Institut international de coopération intellectuelle, 1935, 257 p., p. 103-110, citation p. 103-104.

12. D. C. Röell, «Nouveaux aménagements du Rijksmuseum, Amsterdam », Museum, vol. VIII, n 5 , 1955, Paris, Unesco, 72 p., p. 28-34, citation p. 28. 


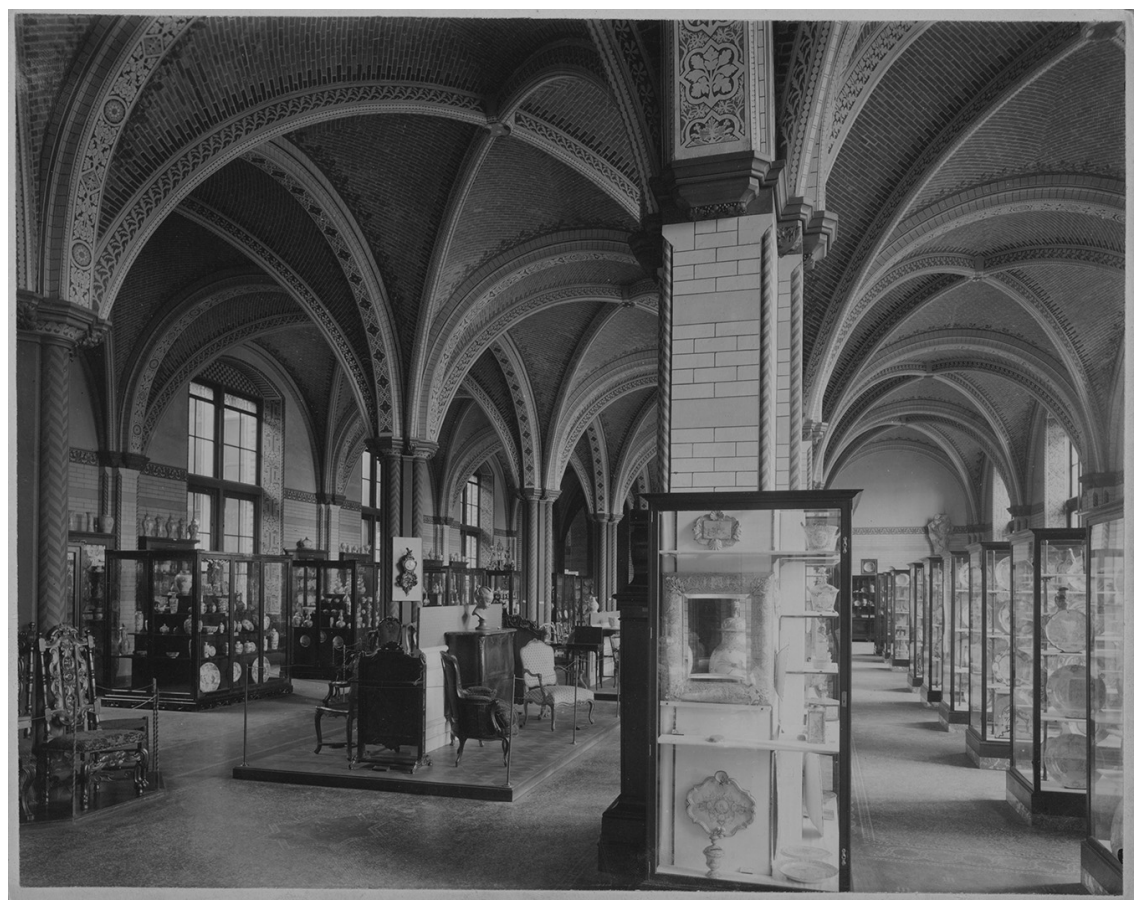

Ill. 1 : Pierre Cuypers, Rijksmuseum (Amsterdam, 1876-1885). La salle des porcelaines, vers 19051909. (C) Courtesy Rijksmuseum, Amsterdam.

Musée, incohérente au-dedans, timide à l'extérieur, établit clairement l'insuffisance de M. Duban, et tous les vrais amis de l'architecture ont le droit de regretter qu'il ait été chargé d'une tâche si délicate ${ }^{13}$. " Le Salon Carré (ill. 2) demeure représentatif de ce vocabulaire ornemental si décrié. Théophile Gautier, quant à lui, proposa qu'on reprenne totalement la voûte de la Grande Galerie: "L'affreux plafond à rosace chicorée n'a rien qu'on puisse regretter ${ }^{14}$ ». En 1895, Gustave Larroumet, de la direction des Beaux-Arts, jugeait de son côté que, pour rendre la visite du Louvre plus agréable, il conviendrait surtout de "gouverner le talent de nos architectes; il faudrait les obliger à subordonner l'installation aux œuvres, à ne pas écraser les tableaux et les statues par l'ornement des plafonds ${ }^{15}$." Henri Verne, ancien directeur des musées nationaux, s'exprima lui aussi sur ces questions lors d'un plan de rénovation du Louvre en 1927-1934: «Depuis 1849, estimait-il, Duban et ses

13. Gustave Planche, Portraits d'artistes : peintres et sculpteurs, Paris, Michel Lévy, vol. II, 1853, 372 p., p. 274.

14. Théophile Gautier, "Étude sur le musée ", Théophile Gautier, Tableaux à la plume, Paris, G. Charpentier, 1880, 336 p., p. 23.

15. Gustave Larroumet, L'Art et l'État en France, Paris, Hachette, 1895, 370 p., p. 237. 


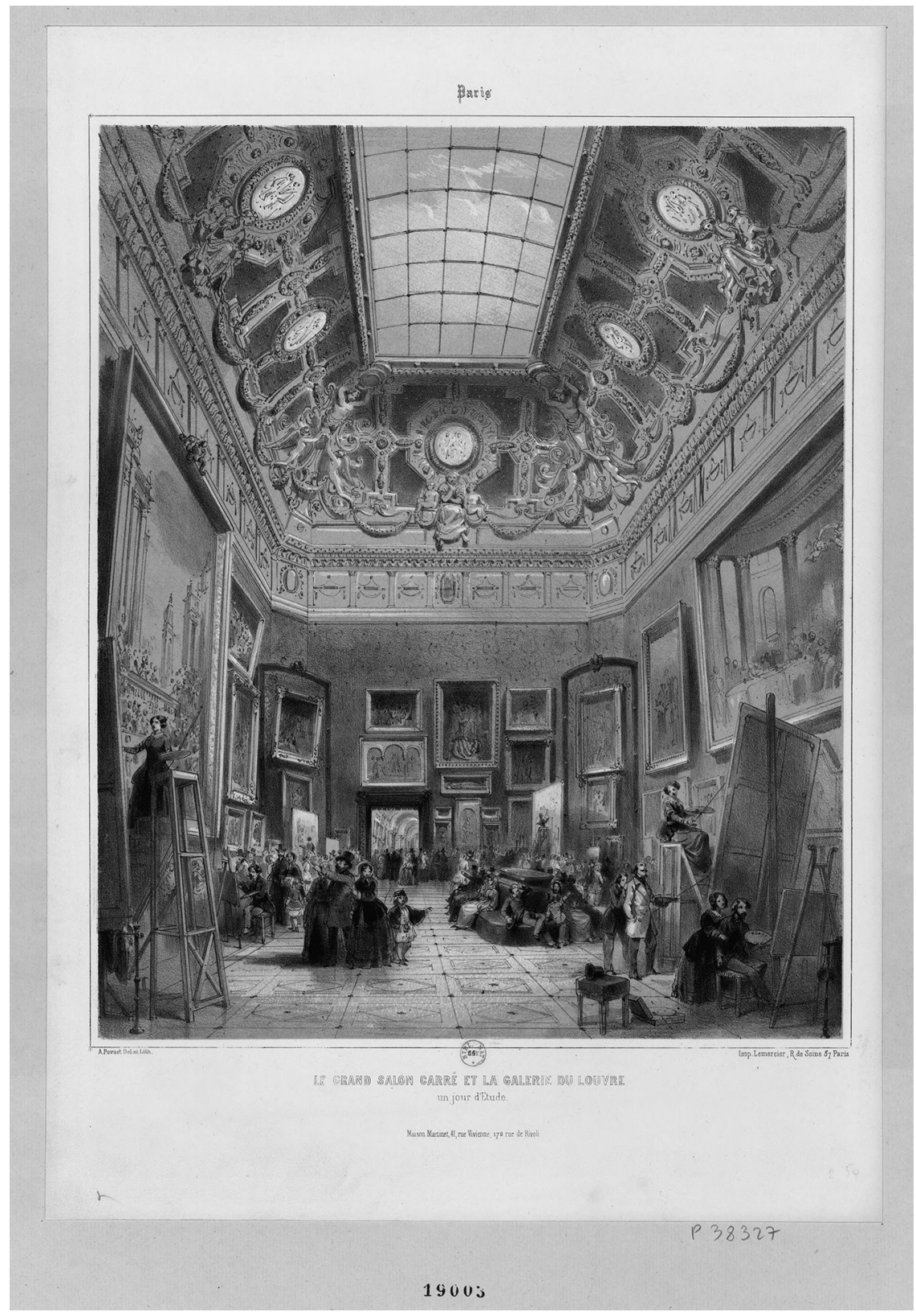

Ill. 2 : A. Provost, "Le Grand Salon Carré et la Galerie du Louvre un jour d'Étude ». Recueil Collection de Vinck. Un siècle d'histoire de France par l'estampe, 1770-1870. Vol. 158 (pièces 18946-19052), Second Empire. Lithographie en camaïeu; $29,6 \times 24,5 \mathrm{~cm}$. 1852. (C) Paris, Bibliothèque nationale de France.

Livraisons d’bistoire de l'architecture $n^{\circ} 30$ 
successeurs ornaient des salles trop hautes de plafonds dorés et sculptés. La tradition des intérieurs trop ornés, dont les œuvres d'art, loin de tirer une mise en valeur, semblaient presque les accessoires, s'est continuée ${ }^{16}$. »

\section{"Les modes peuvent changer"}

Même si Henri Verne parle de "tradition ", l'aménagement des salles, au Louvre particulièrement, est toujours resté sensible aux évolutions des idées en matière d'architecture intérieure. Les décors commandés à Fontaine par Charles X, intégraient ainsi des "plafonds peints par quelques-uns des peintres les plus en vogue ${ }^{17}$ ", garants du succès de la rénovation. Autre époque, autre style avec Lefuel, qui supprima les tons clairs, le gris et l'or pour se mettre «au goût du jour ${ }^{18}$ ». Autour de 1900, l'historicisme se fait sa place, notamment avec Gaston Redon et son décor néo-baroque pour la Galerie Médicis. Dans les années 1930-1950, la tendance fut en revanche de masquer "les décors trop présents ${ }^{19}$ ", en atteste la reprise de la salle des États par Jean-Jacques Haffner, en 1950, et la suppression totale du décor des voussures. L'approche changea encore à la fin des années 1960, lorsque les rénovations consistèrent non plus à créer de nouveaux décors ni à en gommer certains mais à restituer le décor Napoléon III $^{20}$. Pour l'architecte, il faut donc suivre le mouvement, au risque de paraitre anachronique : en 1904, les ornements et les moulures de style Renaissance du décor intérieur du Bode museum, à Berlin, furent jugés dépassés dès l'ouverture du musée ${ }^{21}$. Plus récemment, la reprise des espaces intérieurs du musée d'Orsay par Jean-Luc Wilmotte, moins de vingt-cinq ans après l'intervention de Gae Aulenti, est emblématique de cette versatilité décorative. Aussi Christine Bernier constatait-elle en 2002 que, dans ce domaine comme dans d'autres, "les modes peuvent changer ${ }^{22}$ ".

On se souvient d'ailleurs que Germain Bazin et Michel Laclotte, à quelques années de distance, avaient des vues divergentes en ce qui concerne l'aménagement intérieur du Louvre. Le premier, en 1955, revendiquait "un certain luxe sobre, caractérisé par l'emploi des étoffes de velours, des boiseries, des faux marbres ${ }^{23}$ ".

16. Henri Verne, «Le plan d'extension et de regroupement méthodique des collections du musée du Louvre. Les travaux de 1927 à 1934 ", Bulletin des musées de France, janvier 1934, 40 p., p. 1.

17. Jean-Louis de Cenival, "Antiquités égyptiennes ", Pierre Quoniam, op. cit., p. 64.

18. Christiane Aulanier, La Grande galerie du bord de l'eau, Paris, Réunion des musées nationaux, 1948, 46 p., p. 33.

19. Jean-Luc Martinez, «L'exposition des sculptures antiques au musée du Louvre : histoire d'un malentendu ", Jean-Luc Martinez (ed.), op. cit., p. 211.

20. Georges Bernier, "La rénovation de la Grande Galerie ", L'Eil, n 166, octobre 1968, 84 p., p. $4-13$ et $48-49$.

21. Bernard Schulz, "Treasure island", Speech, "Myzeѝ ", novembre 2013, 288 p., p. 178-197. Voir p. 193.

22. Christine Bernier, L'Art au musée. De l'auvre à l'institution, Paris, L'Harmattan, 2002, 261 p., p. 73.

23. Germain Bazin, "Nouveaux aménagements du département des peintures, Musée du Louvre, Paris ", Museum, vol. VIII, nº 5, op. cit., p. 16-23, citation p. 21-22. 
Le second réclamait plus d'authenticité et déplorait les effets de "mise en scène " (mur en fausse pierre, reconstitution de cabinet de collectionneur, etc.) imaginés par Jean-Charles Moreux et Emilio Terry, les deux architectes-décorateurs auxquels Bazin avait confié le chantier: "C'était une sorte d'évocation-reconstitution au troisième degré ${ }^{24}$ ", raillait-il.

\section{La tentation du pastiche}

L'initiative n'était pourtant pas inédite. Pour abriter le Museo civico de Castelvecchio, en 1923, on avait ainsi procédé à des collages d'éléments néogothiques destinés à donner à l'ensemble l'allure d'un palazzo médiéval ${ }^{25}$. À la fin des années 1950, au musée national d'Athènes, la salle des antiquités mycéniennes avait elle-même été peinte et décorée de façon à évoquer le palais des Atrides ${ }^{26}$. Le musée archéologique de Metz, en 1977, opta quant à lui pour un plafond surbaissé, appuyé sur des murs courbes, pourpre foncé, afin de restituer "l'atmosphère d'un temple souterrain", en accord avec les œuvres exposées ${ }^{27}$. Alexandre Lenoir, dans son musée des monuments français, souhaitait déjà donner "à chacune des salles le caractère, la physionomie exacte du siècle qu'elle doit représenter ${ }^{28}$. " L'idée fut reprise plus tard à la Glyptotek de Munich, où Léo von Klenze fit communiquer l'architecture et son contenu en décorant les salles selon le style des œuvres exposées ${ }^{29}$. Champollion était lui-même très clair lorsqu'on lui confia la création du musée égyptien, en 1826: "Il faut absolument, écrivait-il, que mes salles soient décorées à l'Égyptienne ${ }^{30} "$.

Le principe fit école : Friedrich Stüler, en 1853, imagina ainsi pour les collections égyptiennes du Neue Museum de Berlin un somptueux décor "aux fresques bariolées ${ }^{31}$ ", avec hiéroglyphes et colonnes factices. Au Kunsthistorisches museum de Vienne, dans le dernier quart du XIX ${ }^{\mathrm{e}}$ siècle, l'architecture des salles des antiquités égyptiennes pensée par Gottfried Semper et Carl von Hasenauer s'inspirait des

24. Michel Laclotte, Histoire de musées : souvenirs d'un conservateur, Paris, Scala, 2003, 255 p., p. 97-98.

25. André Gob, Noémie Drouguet, La Muséologie. Histoire, développements, enjeux actuels, Paris, Armand Colin, 2006, 293 p., p. 255.

26. Hans Möbius, "La reconstruction du Musée national d'Athènes ", Museum, vol. XII, n 2, Paris, Unesco, 1959, 63 p., p. 86-94.

27. Gérard Collot, "Ouverture des nouvelles salles au Musée archéologique de Metz ", Musées et collections publiques de France, $\mathrm{n}^{\circ}$ 137, Paris, 1977-1, 47 p., p. 7-11.

28. Cité par Jaap Harskamp dans «Renaissance and renovation. The influence of Lenoir's "Musée des monuments français", 1795-1816 ", Gazette des Beaux-Arts, t. 136, n 1580, septembre 2000, 51 p., p. 103.

29. Peter von Seidlein, "Munich Glyptothek ", The Architectural Review, vol. CLXXI, n 1023 , mai 1982, 86 p., p. 32-38, voir p. 34.

30. Lettres d'Italie, 4 octobre 1826. Cité par Sylvie Guichard (éd.), Notice descriptive des monuments égyptiens du musée Charles X, Paris, Louvre, Khéops, 2013, 368 p., p. 36.

31. Dietrich Wildung, L'Art égyptien à Berlin. Chefs-d'œeuvre du musée Bode et de Charlottenburg, Berlin, SMPK, 1998, 60 p., p. 4. 


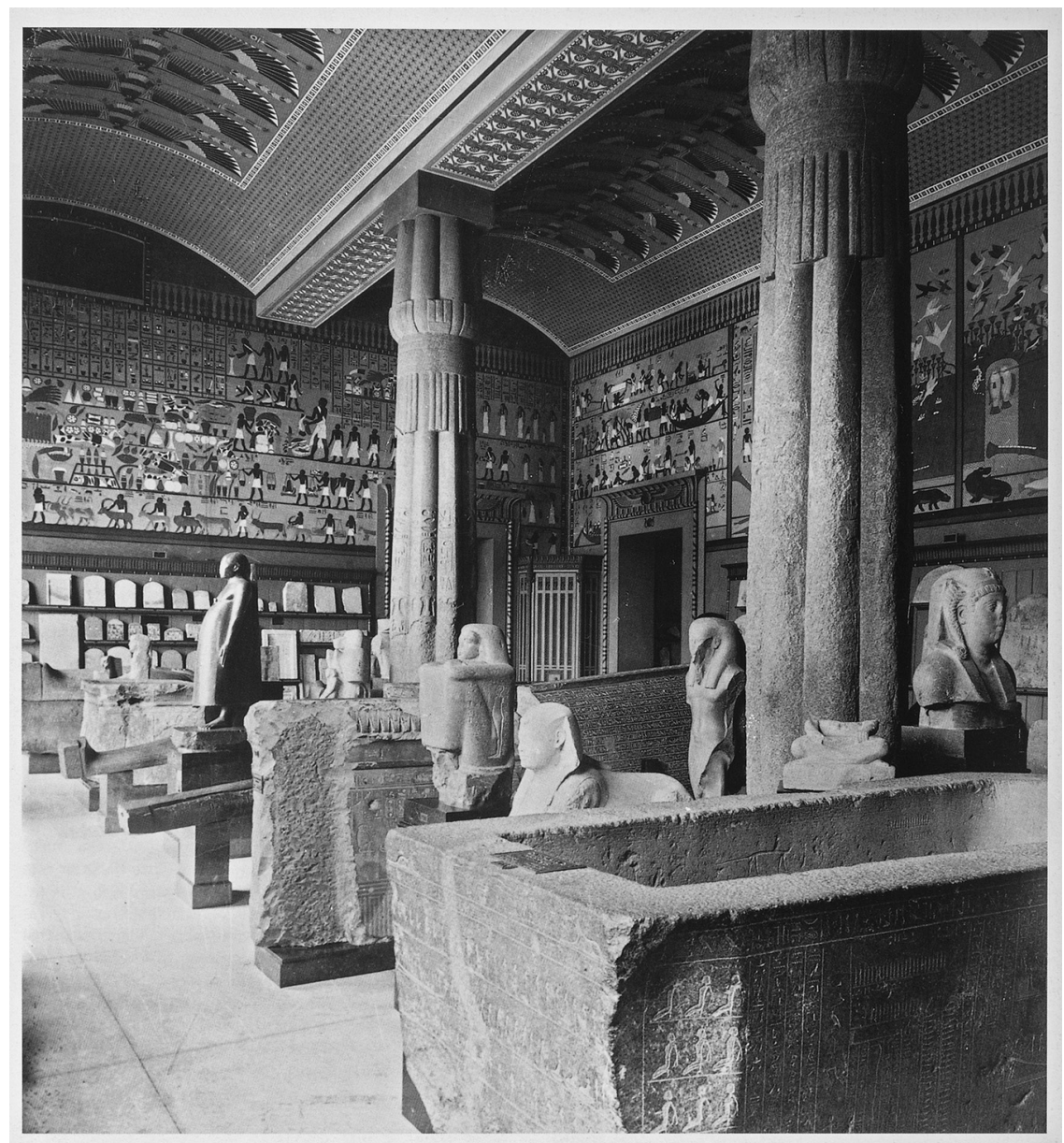

Ill. 3 : Kunsthistorisches Museum, Vienne: la salle des antiquités égyptiennes en 1891. Architectes: Gottfried Semper, Carl von Hasenauer. (C) KHM Museumsverband.

comptes rendus d'une expédition de 1849 (ill. 3) : le plafond voûté, les contours de porte et le décor des murs (couverts de motifs égyptisants dessinés par Ernst Weidenbach) plagiaient l'architecture des anciens temples égyptiens. En 1912, c'est une logique similaire qui avait mené les concepteurs du musée Pouchkine, à Moscou, à doter leur propre salle égyptienne de colonnes lotiformes reprenant celle du temple de Louxor et d'une " ornementation imitant celle des plafonds de l'Égypte antique $^{32} \cdot{ }^{\prime \prime}$

32. S. Hodžaš, V. Deul, "La nouvelle exposition du Département des antiquités égyptiennes et orientales au Musée Pouchkine des beaux-arts, Moscou ", Museum, vol. XXIII, n 4, Paris, Unesco, 1970-1971, 72 p., p. 286-289, citation p. 286. 
Pour sa salle orientale, le musée Pouchkine décida de reproduire certaines décorations assyriennes. Trente ans plus tôt, en 1883, le Louvre avait déjà pris le même parti en chargeant Charles Lameire d'imaginer le décor peint de la " salle assyrienne " du Louvre, ouverte en 1888. On fera usage d'un principe décoratif similaire au Vorderasiatisches museum de Berlin, au début des années 1930, pour agrémenter l'arc du passage constitué par deux sculptures monumentales assyriennes, en l'occurrence deux taureaux androcéphales ailés. Inspirés de relevés archéologiques de la fin des années 1840, ces ornements peints, dont la disposition est par ailleurs correcte, n'en sont pas moins artificiels.

Il n'est certes pas anodin que le projet d'aménagement « dans l'esprit de la fin du $\mathrm{XIX}^{\mathrm{e}}$ siècle $^{33}$ " qui fut retenu pour la récente rénovation de la Wallace Collection, à Londres, ait été taxé de "pastiche ". Qu'à cela ne tienne, l'approche a toujours eu ses militants. Jacques Thuillier, en 1986, regrettait bel et bien que l'on n'ait pas décidé de ressusciter le XIX ${ }^{\mathrm{e}}$ siècle et "le goût de Laloux ${ }^{34}$ " lorsqu'on décida de réhabiliter l'ancienne gare d'Orsay pour en faire un musée. Clarence Samuel Stein, l'architecte du Wichita Art Institute, en 1933, avait en revanche été intraitable sur la question : "Les imitations d'anciennes décorations d'intérieurs sont inadmissibles. Si le visiteur sait qu'il s'agit d'un trucage, il est porté à mettre en doute l'authenticité de tous les objets exposés ${ }^{35}$."

\section{Haro sur le décor}

Trucage ou pas, il est fréquemment reproché au décor d'être trop présent. C'est ce que déplorait Charles J. Holmes, de la National Gallery, en 1928 : «L'ancienne conception de la décoration murale avait imposé des tons riches, vert foncé et rouge, sur un fond rehaussé d'un grand dessin de feuillages (qui) attiraient l'œil au détriment des tableaux ${ }^{36}$. » La salle Van Dyck du Louvre, en 1929, présentait encore ce type de murs revêtus d'une toile peinte au pochoir, à motifs floraux sur fond brun.

De quoi, effectivement, détourner l'attention des visiteurs. Aussi peut-on comprendre que Louis Reckelbus, conservateur au musée de Bruges au début des années 1930, ait estimé que "le bon musée est celui qui n'existe pas pour lui-même mais pour les œuvres. Il est inutile que le visiteur s'intéresse au ton des parois ${ }^{37}$.

33. Maureen Marozeau, "La Wallace collection prête pour le XXe siècle ", Le Journal des Arts, $\mathrm{n}^{\circ} 419$, 19 septembre-2 octobre 2014, 40 p., p. 7.

34. Jacques Thuillier, "De la gare au Musée d'Orsay", Revue de l'Art, 1986, nº 74, 77 p., p. 5-11, citation p. 7.

35. Clarence S. Stein, "Architecture et aménagement des musées », Mouseion, vol. 21-22, no 1-2, Paris, Institut international de coopération intellectuelle, 1933, 290 p., p. 7-26, citation p. 12.

36. Sir Charles J. Holmes, "Les transformations de la "National Gallery" à Londres ", Mouseion, n 4 , Paris, Institut international de coopération intellectuelle, avril 1928, 261 p., p. 1-6, citation p. 2.

37. Paul Fierens, "Le Musée communal de Bruges ", Mouseion, vol. 29-30, Paris, Institut international de coopération intellectuelle, 1935, 258 p., p. 213-236, citation p. 219. 
Il fallait, selon ses dires, "que le musée s'oublie ». Et le résultat était en effet austère, comme en témoigne le commentaire de l'époque sur les salles de la peinture du $\mathrm{XV}^{\mathrm{e}}$ siècle : "À gauche part une enfilade d'alvéoles communiquant entre elles par des ouvertures en plein cintre [...] Un bandeau de briques roses agrémente délicatement le contour des portes. Nul autre ornement n'est admis dans cette partie du musée ". À la même période, Leo van Puyvelde, conservateur aux musées royaux des Beaux-Arts de Belgique, estimait sans détour " [qu']il est de première importance de donner aux parois contre lesquelles on place des objets, une tonalité neutre et claire. Les fonds portant des décorations nuisant à l'aspect des œuvres d'art ${ }^{38}$. " Le débat était d'ailleurs ancien : lorsque, en raison de la grande guerre, la décoration intérieure du Science Museum de Londres ne put être réalisée, on avait finalement conclu que "dans cet état inachevé et sans décoration, l'édifice offrait un décor certainement plus heureux pour les objets à exposer ${ }^{39}$. " Du reste, le rapport des conservateurs du musée du Louvre, le 13 décembre 1794, se montrait déjà intransigeant à ce sujet : "La décoration intérieure doit se réduire à séparer par des colonnes les objets d'art ou les écoles ${ }^{40}$ ".

Il s'agirait donc, un tant soit peu, d'en finir avec le décor. Marcel Proust, on peut s'en souvenir, avait en son temps milité en faveur de la "nudité " et du "dépouillement " de la salle de musée ${ }^{41}$. Louis Hautecœur, en 1933, précisait que la présence des collections suffit et rend inutile toute ambition décorative : "Dans un musée moderne, écrivait-il, l'architecte doit renoncer au décor pour le décor : ce sont les objets d'art eux-mêmes qui ornent le musée ${ }^{42}$. " Martin Wagner, en Allemagne, dans les années 1920, soutenait déjà un édifice purement fonctionnel, sans ornement. En Allemagne, toujours, mais bien plus tard, Detlef Hoffman appréciait que dans son musée romano-germanique de Cologne, le choix ait été de faire disparaître le décor en le plaçant dans l'obscurité ${ }^{43}$. Récemment, Frédéric Bonnet, dans le Journal des arts, prisait aussi l'absence de "concession faite à l'ornementation " au musée de Bolzano ${ }^{44}$ et Renaud Piérard, l'architecte chargé de la muséographie du département des arts de l'Islam, au Louvre, arguait que son projet ne

38. Leo van Puyvelde, "Principes de la présentation des collections dans les musées ", Mouseion, vol. 25-26, Paris, Institut international de coopération intellectuelle, 1934, 235 p., p. 36-43, citation p. 40.

39. John H. Markham, "Le plan et la conception architecturales de musées ", Mouseion, vol. 29-30, op. cit., p. 7-22, citation p. 8-9.

40. Cité par Christiane Aulanier, La Grande galerie du bord de l'eau, op. cit., p. 16.

41. Voir À l'ombre des jeunes filles en fleurs, Paris, Éditions de la Nouvelle Revue française, 1920, 250 p., p. 197.

42. Louis Hautecœur, "Architecture et organisation des musées ", Mouseion, vol. 23-24, Paris, Institut international de coopération intellectuelle, 1933, 257 p., p. 5-29, citation p. 14.

43. Detlef Hoffmann, "Problème d'une conception didactique du musée », André Desvallées (éd.), Vagues. Une anthologie de la nouvelle muséologie I, Mâcon, Éditions W., Savigny-le-Temple, MNES, 1992, 529 p., p. 387-404.

44. Frédéric Bonnet, "Un Museion en transition», Journal des Arts, n 423, 14 novembre 2014, 40 p., p. 14. 
comportait "pas de design superflu ${ }^{45}$. " Le risque étant, bien sûr, d'en arriver à un anonymat qui peut faire polémique. Laurent Wolf se montrait volontiers critique en 2004 : "Rien ne ressemble plus à un centre d'art français qu'une Kunsthalle allemande. Rien ne ressemble plus, malgré les prouesses des architectes, aux salles d'un musée d'art contemporain d'ici que celles d'un musée d'art contemporain d'ailleurs ${ }^{46}$."

\section{Du compromis à la confusion}

Dès 1934, Paul Cret avait jugé bon de prendre parti contre ce qu'il appelait "la vogue actuelle des salles dépourvues de toute ornementation ${ }^{47}$. "Son opinion était simple: "L'architecture dans le musée n'a pas à jouer le rôle de parente pauvre." D'un côté, il faut renoncer à ce qu'il décrit comme "les murs antiseptiques du musée idéal ", de l'autre il convient de faire preuve de modération. Il résumait ainsi la situation: "S'il ne faut pas faire un "faux" temple égyptien ou un "faux" salon Louis XIV, il ne faut pas non plus que les meubles de Boulle aient l'air d'attendre le déménageur."

Un compromis est donc suggéré, en déclinant par exemple le décor du musée en regard des œuvres d'art. Le lien peut en premier lieu être iconographique. À l'ouverture du Musée des Antiques, fin 1800, les sculptures présentées dans la salle des Saisons et dans la salle des Hommes illustres furent ainsi choisies en regard des décors du plafond. Le lien peut aussi être plus ténu, comme au musée de Valladolid, dans le premier tiers du $\mathrm{XX}^{\mathrm{e}}$ siècle, où l'aspect des murs avait été pensé en écho aux statues polychromées de la collection ${ }^{48}$. Le projet rejoignait celui du musée d'art de Catalogne, en 1935, comme le raconte le directeur de l'époque : "En ce qui concerne le caractère des aménagements ou, pour mieux dire, le "style" des installations, on ne s'est pas écarté du principe suivant lequel la fonction primordiale du musée est la mise en valeur des objets exposés. C'est pourquoi les lignes, les surfaces, les couleurs et les matières qui forment l'ambiance des œuvres d'art, ont été choisies en se basant sur la nature des objets - d'après leurs formes, leur caractère et leur aspect matériel ${ }^{49}$. " Henri Van de Velde, en 1902, avait déjà pensé le décor intérieur du Museum Folkwang, à Hagen, en adéquation complète avec les œuvres présentées, sur le modèle conceptuel de l'œuvre d'art totale ${ }^{50}$.

45. Véronique Prat, "L'islam en plein art ", Le Figaro, 14 septembre 2012. En ligne : http://www.lefigaro. fr/arts-expositions/2012/09/14/03015-20120914ARTFIG00761-1-islam-en-plein-art.php?pagination=5

46. Laurent Wolf, Vie et mort du tableau. 1273-1973. 1. Genèse d'une disparition, Paris, Klincksieck, 2004, 170 p., p. 13.

47. Paul P. Cret, "L'architecture des musées en tant que plastique ", Mouseion, vol. 25-26, op. cit., p. 7-16, citation p. 15 .

48. F.-J. Sanchez Canton, "Le nouveau musée national de sculpture de Valladolid ", ibid., p. 84-105.

49. Joaquim Folch y Torres, "La nouvelle installation du Musée d'Art de Catalogne ", Mouseion, vol. 31-32, op. cit., p. 61-66, citation p. 64 .

50. Voir Katherine Kuenzli, "The Birth of the Modernist Art Museum : The Folkwang as Gesamtkunstwerk ", Journal of the Society of Architectural Historians, vol. 72, n 4, décembre 2013, 187 p., p. $503-529$. 
Avec, en bout de course, le risque de confondre les collections et l'environnement architectural, sur le modèle de la Villa Albani, où les reliefs antiques étaient insérés dans le décor d'ensemble. Les collections privées du XVII ${ }^{e}$ siècle maintenaient déjà une certaine ambiguïté entre les œuvres de la collection et les objets de décor, du moins dans certaines parties des lieux ${ }^{51}$. Quitte, parfois, à être un brin expéditif, comme au musée de Vienne au début du VIII siècle : "Pour les faire entrer à tout prix dans la "décoration" on n'a pas craint de mutiler les œuvres, de les amputer ou leur ajouter des "compléments" 52 ", constate Vinzenz Oberhammer. On peut aussi se souvenir, dans un registre plus léger, de ce qu'écrivait Paul-Émile Botta à Jules Mohl en 1844 au sujet des sculptures monumentales découvertes sur le site de Khorsabad, en Assyrie: "Elles feront une belle porte pour le musée ${ }^{53}$."

Yannick LE PAPE ancien élève de l'École normale supérieure de Cachan, docteur en histoire et civilisations (EHESS), ingénieur des services culturels et du patrimoine au musée d'Orsay

51. Christophe Piccinelli-Dassaud, "Collectionner des marbres antiques en France au XVII ${ }^{\mathrm{e}}$ siècle ", Jean-Luc Martinez (éd.), op. cit., p. 13-20.

52. Vinzenz Oberhammer, La peinture au musée de Vienne, Paris, Éditions Cercle d'art, 1965, non pag.

53. Cité par Élisabeth Fontan, "Adrien de Longpérier et la création du musée assyrien du Louvre ", Élisabeth Fontan, Nicole Chevalier (dir.), De Khorsabad à Paris, Paris, RMN, 1994, 286 p., p. 226-239, p. 226. 
